\title{
Triple Wollaston-prism complete-Stokes imaging polarimeter
}

\author{
John D. Perreault ${ }^{1,2}$ \\ ${ }^{1}$ Visible and Infrared Sensor Systems Department, The Aerospace Corporation, 2310 E. El Segundo Blvd., \\ El Segundo, California 90245-4609, USA \\ ${ }^{2}$ Now with Google[x], Google, Inc., 1600 Amphitheatre Parkway, Mountain View, California 94043, USA (john.d.perreault@aero.org)
}

Received June 13, 2013; revised August 13, 2013; accepted August 21, 2013;

posted August 21, 2013 (Doc. ID 192231); published September 26, 2013

\begin{abstract}
Imaging polarimetry is emerging as a powerful tool for remote sensing in space science, Earth science, biology, defense, national security, and industry. Polarimetry provides complementary information about a scene in the visible and infrared wavelengths. For example, surface texture, material composition, and molecular structure will affect the polarization state of reflected, scattered, or emitted light. We demonstrate an imaging polarimeter design that uses three Wollaston prisms, addressing several technical challenges associated with moving remote-sensing platforms. This compact design has no moving polarization elements and separates the polarization components in the pupil (or Fourier) plane, analogous to the way a grating spectrometer works. In addition, this concept enables simultaneous characterization of unpolarized, linear, and circular components of optical polarization. The results from a visible-wavelength prototype of this imaging polarimeter are presented, demonstrating remote sensitivity to material properties. This work enables new remote sensing capabilities and provides a viable design concept for extensions into infrared wavelengths. (c) 2013 Optical Society of America

OCIS codes: (110.5405) Polarimetric imaging; (280.0280) Remote sensing and sensors; (070.0070) Fourier optics and signal processing.

http://dx.doi.org/10.1364/OL.38.003874
\end{abstract}

Light has several physical properties that are useful for remote sensing applications in space science, Earth science, biology, defense, national security, and industry. The intensity and wavelength of light has been used widely to gain spatial and spectral information from a scene. However, polarization has not been as extensively investigated for imaging applications, although some exciting applications are starting to be explored. Polarimeters can be used in such diverse areas as passive remote sensing [1] for the detection of improvised explosive devices [2], characterization of aerosol particles in the Earth atmosphere [3], extra-solar planet detection [4], remote mapping of planetary magnetospheres [5], quality control for welding [6], and the search for biological activity on other planets [7]. There are even some animals, such as the mantis shrimp, that have evolved sophisticated polarimetric visual systems to obtain valuable information from their environment, and are sensitive to all the polarization components of light [].

These applications stem from the basic interaction of light with a solid surface, liquid surface, or gas. The lightmatter interactions alter the amplitude and phase of polarization states in a way that depends on material properties. For example, conductive materials have a complex index of refraction that will cause a phase-shift between orthogonal polarization states, generating elliptically polarized light [9]. This effect can provide contrast between conductive and nonconductive matter, enabling material discrimination and artificial object detection. The total degree of polarization is also affected by the texture and topology of a surface, and requires complete knowledge of all the polarized and unpolarized components for unambiguous characterization. In addition, all known biological systems have evolved to prefer a particular chirality (or handedness) of organic molecules. This asymmetry in the occurrence of stereoisomers can lead to a unique polarization signal through circular dichroism or optical activity [7,10]. In order to exploit these phenomena one needs to build a polarimeter that can measure both linear and circular polarization, using a robust design that is suitable for a moving platform. In this Letter we put forth a new scanning-polarimeter design that simultaneously measures all the polarization components without moving polarization elements, and demonstrate its operation in the visible wavelengths.

There are several technical challenges to measuring polarization that need to be addressed in the context of moving remote-sensing platforms. The design of a flight-ready imaging polarimeter must be simple, compact, and robust over a range of environmental conditions. Existing designs use wire-grid polarizers placed on top of a specialized focal-plane array (FPA), complex arrangements of beam-splitters and polarization elements, or moving parts. The wire-grid polarizers are positioned in the image plane and need to be aligned to the FPA much better than a pixel to minimize cross-talk [1]. Any vibration of the image makes it difficult to separate polarization and intensity changes. Complex optical systems that need to be carefully aligned, or that have moving parts, are not ideal for launch or flight conditions. Polarimeters that utilize photo-elastic modulators have overcome some of these challenges, but the components are fragile and will not operate properly at cryogenic temperatures required for infrared wavelengths [11].

A solution to these problems is a polarimeter design based on Wollaston prisms that induces an angular displacement of light in the Fourier (i.e., pupil) plane in a way that depends on the polarization state. This concept is analogous to the way a grating spectrometer induces an angular displacement that depends on wavelength $[10,12]$. Simple linear polarimeters based on Wollaston prisms have an impressive history and were used to measure two orthogonal polarization states on groundbreaking missions, such as the Pioneer spacecraft [13]. Later 

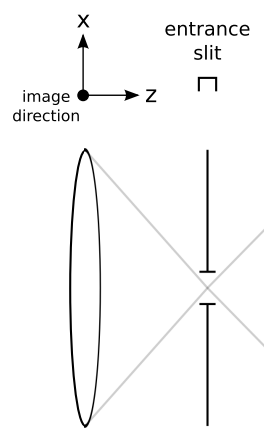

L1

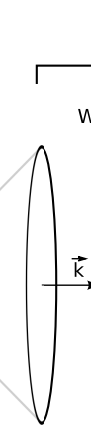

L2
Fourier

space

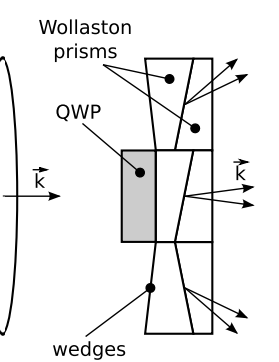

WA image

plane

$\sqcap$

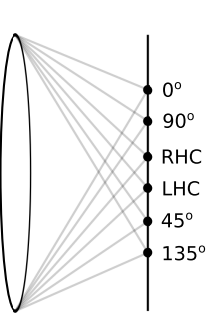

L3
FPA
Fig. 1. Diagram of the triple-Wollaston polarimeter. An image from lens L1 is projected onto the entrance slit SL1, with the image-direction coming out of the page. The entrance slit is placed at the front-focal plane of lens L2, collimating the light. The Wollaston-prism assembly (WA) induces a small angular displacement (i.e., rotation of $\vec{k}$ ) that depends on the polarization state, and the wedges separate the outputs. The projections of linear $\left(0^{\circ}, 90^{\circ}, 45^{\circ}, 135^{\circ}\right)$, right- (RHC), and left-hand-circular (LHC) polarizations are re-imaged onto the focal-plane array (FPA) by lens L3. A two-dimensional image of the complete polarization state (Stokes vector) is acquired by scanning the image across SL1 in the $x$ direction.

designs proposed a double-Wollaston prism that could measure four nonorthogonal projections, completely characterizing the linear polarization of light [14]. However, all of these approaches measure only the linear component of polarization.

Our new triple-Wollaston imaging-polarimeter design, as described in Fig. 1, completely characterizes the entire polarization state for each point in an image. The basic design concept is a slit-scanning optical-relay system with the triple Wollaston-prism assembly (WA) placed in the pupil plane. The system consists of a front-end telescope $\mathrm{L} 1$ projecting a $2 \mathrm{D}$ image onto a $25 \mu \mathrm{m} \times 25.4 \mathrm{~mm}$ chrome-on-glass slit SL1 manufactured by Lenox Laser. The slit-image is optically relayed by L2, L3 to a $12 \mu \mathrm{m}$ pixel DALSA Pantera 6M8 camera FPA after being polarimetrically analyzed by WA. The intensity of each polarization projection on the FPA in Fig. 1 is related to the Stokes vector

$$
\mathbf{S}=\left[\begin{array}{c}
s_{0} \\
s_{1} \\
s_{2} \\
s_{3}
\end{array}\right] \propto\left[\begin{array}{c}
I_{0}+I_{90} \\
I_{0}-I_{90} \\
I_{45}-I_{135} \\
I_{\mathrm{LHC}}-I_{\mathrm{RHC}}
\end{array}\right]
$$

The lenses L1, L2, L3 were Nikkor $105 \mathrm{~mm} f / 2.5$ fiveelement commercial camera objectives. The elements of the polarimeter are coupled together with the 2 " Thorlabs lens-tube system to control stray-light, and are mounted on a light-weight rigid optical-rail for mobility. The system dimensions are approximately $0.1 \times 0.2 \times$ $0.5 \mathrm{~m}$. The $2 \mathrm{D}$ image was scanned across SL1 at a $7 \mathrm{~Hz}$ FPA-limited line-rate using a DC motor, linear stage, and a Newport GPIB motion-control system to translate L1 in the $x$ direction. However, the scanning could be accomplished using the remote sensing platform motion itself, eliminating the need for any moving parts in the polarimeter.

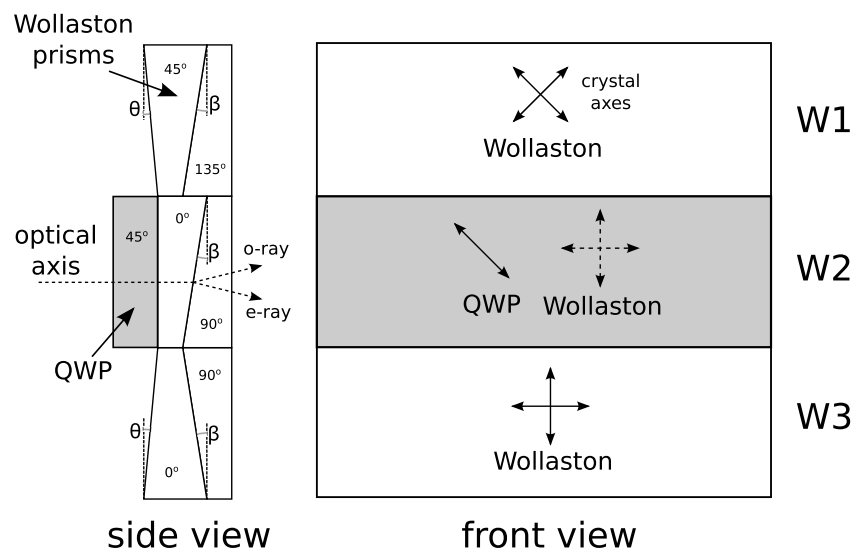

Fig. 2. Details of WA referenced in Fig. 1. The assembly consists of birefringent uniaxial-crystal wedges (white) grouped into prism pairs $\mathrm{W} 1, \mathrm{~W} 2, \mathrm{~W} 3$, along with a zero-order QWP (gray). The principal axis of the QWP is oriented at $45^{\circ}$ with respect to the axes of $\mathrm{W} 2$, the principal axes of $\mathrm{W} 1$ and $\mathrm{W} 3$ are displaced by $45^{\circ}$, and all principal axes are within the $x-y$ plane. The angular deviation between orthogonal pairs of polarization states is determined by $\beta$, while $\theta$ determines the separation between the outputs of each prism pair.

The key-enabling component in the polarimeter design is the triple WA, as shown in Fig. 2. We designed the prism assembly WA, and the $25.4 \times \overline{2} 5.4 \times 8.5 \mathrm{~mm}$ optic was custom-fabricated from $\mathrm{MgF}_{2}$ by Karl Lambrecht Corporation. Magnesium fluoride was chosen for its broad transmission (UV to mid-wave infrared) and small birefringence, which made prism angles more practical [15]. All incidence angles were chosen to be relatively small in order to minimize Fresnel, dispersion, and aberration effects in the polarimeter. Crystalline $\mathrm{MgF}_{2}$ is a uniaxial birefringent material. For small angles, the WA induces an angular deviation $2 \beta \Delta n$ between orthogonal polarization states and $\theta\left(n_{\text {avg }}-1\right)$ between prism-pair outputs, where $\Delta n \equiv n_{e}-n_{o}$ and $n_{\text {avg }} \equiv\left(n_{e}+n_{o}\right) / 2$. The values of $\beta=8.81^{\circ} \pm 0.17^{\circ}$ and $\theta=1.09^{\circ} \pm 0.17^{\circ}$ were chosen to ensure sufficient polarization channel separation on the FPA. Optical-contact bonding (i.e., van der Waals force) was used to construct the Wollaston-prism pairs, so that the transmission properties of $\mathrm{MgF}_{2}$ were not limited by optical cement [16]. $\mathrm{A} \mathrm{MgF}_{2}$ zero-order quarter-wave plate (QWP) was optically contacted to W2 and designed for a central wavelength of $632.8 \mathrm{~nm}$ $[\underline{10}, \underline{15}]$. The prisms and waveplate were cut out of the bulk material so that the crystal axis orientations are given by Fig. 2, enabling measurement of all polarization projections necessary to obtain the full set of Stokes parameters [10].

There are several advantages inherent to the tripleWollaston polarimeter design. The slit SL1 ensures spatial coherence along the $x$ direction. This means that for a sufficiently small wavelength range we can regard the polarimeter as a 4- $f$ Fourier optics system. Therefore, the WA acts as a phase-only polarization Fourier-hologram by separating the polarization components in the spatial-Fourier (pupil) plane. This results in reduced sensitivity to misalignment, less vibration sensitivity, simultaneous measurement of polarization components, minimal cross-talk between polarization channels, greater efficiency compared to absorbing polarization 
FPA Images:

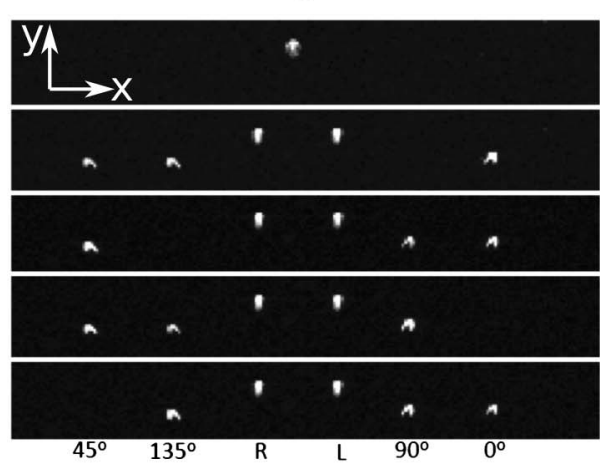

input polarization

state:
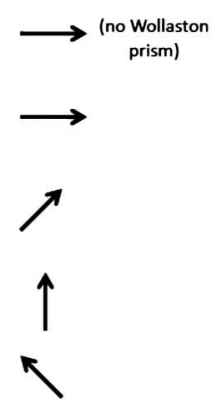

FPA Images:

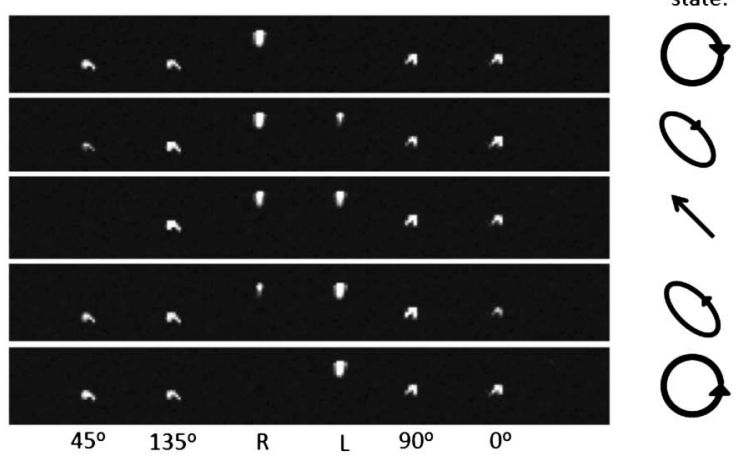

Fig. 3. Demonstration of triple Wollaston-prism WA operation. The images show the response of the system to a point source when varying the linear polarization angle (left) and ellipticity (right). The FPA images are arranged to show the polarization projections along columns and the input polarization state along the rows. The upper-left image shows the system response when WA is removed. Some defocus was introduced in order to increase the spot-size and minimize pixel boundary effects. The lack of signal in the projections orthogonal to the input state indicates exceptional crosstalk performance.

elements, and unambiguous separation of intensity and polarization measurements on the FPA. Much of the alignment robustness of this design comes from the fact that a displacement in the Fourier plane simply causes a global phase shift on the FPA, and is inconsequential since we are measuring intensity at the detector [12]. From the provided angle tolerances $\sigma=0.17^{\circ}$ we can use Malus' law to estimate that WA has an extinction ratio of $1 / \sin ^{2} \sigma \approx 10^{5}$, but the system is currently limited to $\sim 10^{3}$ due to stray-light and FPA performance. However, this is considerably better than the extinction ratio of $\sim 10^{2}$ for wire-grid polarizers.

An example of the output from WA is shown in Fig. 3. The system in Fig. 1 was tested by removing L1, SL1, Lי2 and illuminating $\mathrm{WA}$ with highly collimated light from a $632.8 \mathrm{~nm}$ He-Ne laser. The input polarization state was adjusted using a high-quality linear-polarizer, QWP, and half-wave plate. It is clear that each polarization state produces a unique spatial intensity pattern. When varying the angle of linear polarization, one can see that the projection orthogonal to the polarization state disappears, while the circular projections remain constant.
Correspondingly, when the ellipticity is varied the orthogonal circular polarization vanishes. We also observed that unpolarized light caused all of the spots to have equal relative intensity, as expected. It is important to note that the spots should be colinear along the $x$ direction, but there is a small displacement of the circular polarization components. The displacement is consistent with an erroneous $0.22^{\circ}$ wedge on the central prism pair W2, slightly out of the specified tolerance of $0.17^{\circ}$. However, this displacement can be corrected in postprocessing by spatially shifting the circular polarization images back into alignment with the other polarization channels. These tests confirmed that the crystal axes are oriented properly in each of the Wollaston prisms, and that the zero-order QWP was fabricated correctly.

After the WA operation was verified, the imaging polarimeter was used to look at various targets that were expected to show interesting polarization phenomenon. Figure $\underline{4}$ displays the polarimeter images of a stainlesssteel razor-blade that is partially covered by vinyl electrical tape. The razor is illuminated by polarized light oriented so that there is roughly equal projections of
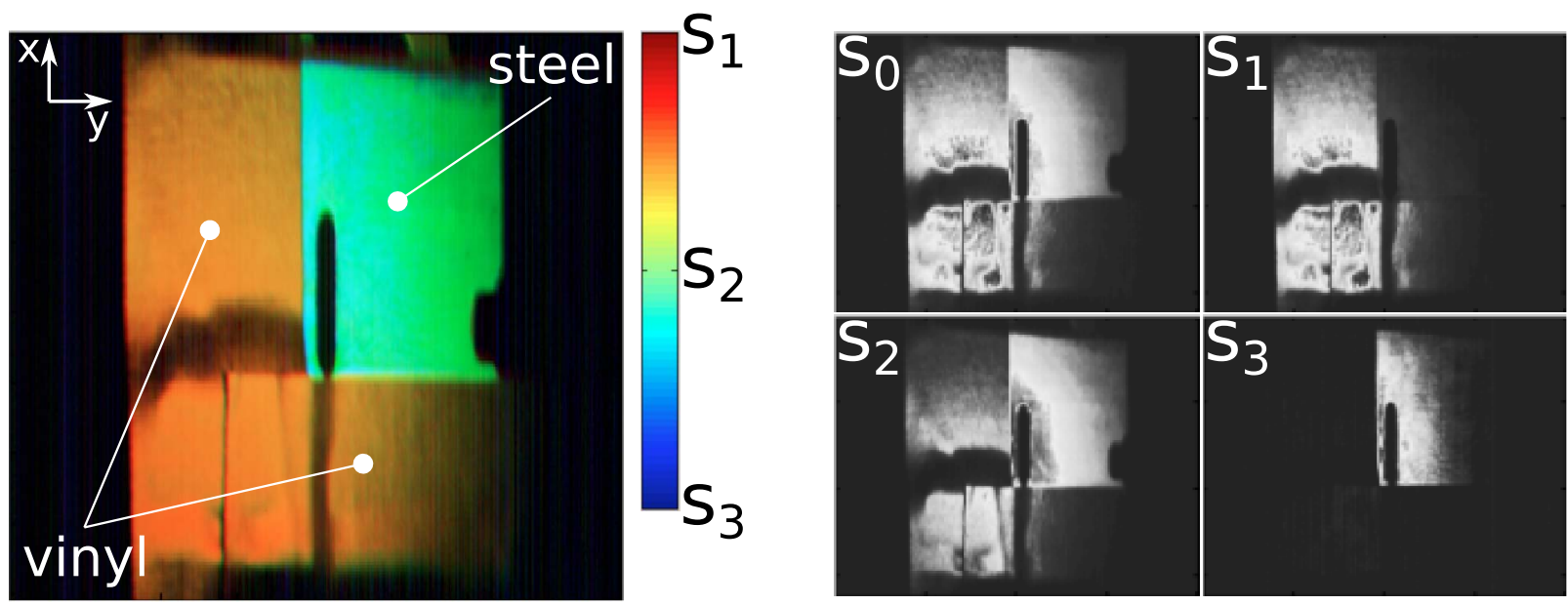

Fig. 4. Polarization image (cropped) of a tilted stainless-steel razor blade partially covered with vinyl tape. The razor blade is illuminated and viewed by the polarimeter at approximately $45^{\circ}$ incidence angle. In both the composite (left) and normalized Stokes magnitude (right) images one can see that the $s_{3}$ component has almost completely vanished for the vinyl tape. This is a clear demonstration of material discrimination between conductive and nonconductive materials. 
$s$ - and $p$-polarization. The raw-image that is acquired contains the polarization projections indicated on the FPA in Fig. 1 and Eq. (1), and six 1D images are extracted by integrating each channel along the $x$ direction. The image is then scanned across slit SL1 to build up the 2D polarization images. One can clearly see that the vinyl electrical tape has a very small $s_{3}$ component compared to the steel. This observation is consistent with the complex index of refraction for conductors causing a phase-shift between $s$ - and $p$-polarizations [9].

In conclusion, an imaging-polarimeter design concept has been put forth, and an experimental prototype was demonstrated. This imaging-polarimeter completely characterizes the polarization of light, by measuring circular and linear components. The design contains no moving polarization elements, does not require a specialized FPA, and is suitable for use from a moving remote-sensing platform. This polarimeter opens up remote-sensing applications in space science, Earth science, biology, defense, national security, and industry. Unique material discrimination and contrast enhancement features are enabled by the complete polarization measurements that this polarimeter provides. The tripleWollaston design is a good candidate for mid-wave infrared polarimetry due to the remarkable transmission and dispersion properties of $\mathrm{MgF}_{2}$ [티] . In addition, longwave infrared designs for WA could be fabricated from CdS or CdSe [17] and applied to investigate emissive polarimetry $[1,2,18]$. However, it is unknown if the $\mathrm{MgF}_{2}$ optical contact bonds can survive the cryogenic thermal cycling required for IR operation, possibly requiring alternative mounting schemes. In the future, it may be possible to replace the Wollaston prism with a polarization hologram [19]. A dispersive grating could be inserted between WA and L3 to add spectral sensitivity along the $x$ direction, while ensuring that the polarization projections do not overlap.
The author acknowledges useful discussions with John Hackwell, David Warren, Bruce Lambert, and Thomas Hayhurst and experimental support from David Berneir, Adam Vore, and Jonathan Bray.

\section{References}

1. J. S. Tyo, D. L. Goldstein, D. B. Chenault, and J. A. Shaw, Appl. Opt. 45, 5453 (2006).

2. K. P. Gurton and M. Felton, Opt. Express 20, 22344 (2012).

3. M. I. Mishchenko and L. D. Travis, J. Geophys. Res. 102, 16989 (1997).

4. S. V. Berdyugina, A. V. Berdyugin, D. M. Fluri, and V. Piirola, Astrophys. J. 673, L83 (2008).

5. L. Ben-Jaffel, W. Harris, V. Bommier, F. Roesler, G. E. Ballester, and J. Jossang, Icarus 178, 297 (2005).

6. N. Coniglio, A. Mathieu, O. Aubreton, and C. Stolz, Opt. Lett. 38, 2086 (2013).

7. W. B. Sparks, J. H. Hough, L. Kolokolova, T. A. Germer, F. Chen, S. DasSarma, P. DasSarma, F. T. Robb, N. Manset, I. N. Reid, F. D. Macchetto, and W. Martin, J. Quant. Spectrosc. Radiat. Transfer 110, 1771 (2009).

8. N. W. Roberts, T. H. Chiou, N. J. Marshall, and T. W. Cronin, Nat. Photonics 3, 641 (2009).

9. M. Born and E. Wolf, Principles of Optics (Cambridge University, 1999).

10. E. Hecht, Optics (Addison-Wesley, 1998).

11. W. Sparks, T. A. Germer, J. W. MacKenty, and F. Snik, Appl. Opt. 51, 5495 (2012).

12. J. W. Goodman, Introduction to Fourier Optics (McGrawHill, 1996).

13. S. F. Pellicori, E. E. Russell, and L. A. Watts, Appl. Opt. 12, 1246 (1973).

14. E. Oliva, Astron. Astrophys. Suppl. Ser. 123, 589 (1997).

15. M. J. Dodge, Appl. Opt. 23, 1980 (1984).

16. H. I. Smith, J. Acoust. Soc. Am. 37, 928 (1965).

17. D. B. Chen and R. A. Chipman, Appl. Opt. 32, 4223 (1993).

18. A. G. Worthing, J. Opt. Soc. Am. 13, 635 (1926).

19. F. Gori, Opt. Lett. 24, 584 (1999). 